\title{
DIDACTICISMO: DILACIÓN SOBRE EL ESTUDIO DEL SABER ENSEÑAR, EN LA FORMACIÓN DOCENTE*
}

Prfsr. Mg. Juan Manuel Negrelli**

Resumen: Los hallazgos de una investigación sobre la formación didáctica de los profesores de Educación Física, propuesta por la transformación curricular reciente en una institución formadora, sensibiliza la atención sobre el saber enseñar en la teoría didáctica. El didacticismo, como se ha categorizado al proceso curricular en estudio, denota una dilación sobre el estudio del saber enseñar. Se presentan a partir de ello algunas vinculaciones y reflexiones entre la transformación curricular, algunas repercusiones en el carácter profesional con que se desarrolla la docencia y la posibilidad de que dichos asuntos constituyan una política de estado.

Palabras claves: Formación de profesores. Didáctica específica. Saber enseñar. Didáctica general.

Resumo: Os achados de uma investigação na formação didática dos professores de Educação Física, sobre as alterações curriculares propostas pelo mais recente de uma instituição de formação, sensibiliza a atenção no saber ensinar na teoria didática. O didaticismo, como classificado pelo processo curricular em estudo, denota um atraso no estudo de aprender a ensinar. Apresentamos algumas ligações e reflexões entre as mudanças de currículo, impactos sobre o desenvolvimento do ensino no seu caráter profissional e da possibilidade de tais questões possam constituir uma política de estado. 
Palavras-chave: Formação de professores. Didática específica. Saber ensinar. Didática geral.

\begin{abstract}
The findings of an investigation into the didactical training of physical education teachers, about curriculum changes proposed by the latest in a training institution, sensitizes attention in teaching knowledge didactic theory. The didacticism, as categorized by curriculum process under study, denotes a delay on the study of learning to teach. We present it from some links and reflections between curriculum changes, some impact on the character that develops professional teaching and the possibility that such matters constitute a state policy.
\end{abstract}

Keywords: Teacher Training. Specific Didactics. Knowing teach. Didactic. 


\section{Introducción}

La posibilidad de que la afirmación "la educación es una ocupación de dudosa importancia” fuera tomada en serio en alguna sociedad contemporánea, gravita entre una en mil. Junto a salud, vivienda y trabajo, la educación es un pilar imprescindible para sociedades que pretenden desarrollarse según algunos parámetros que la modernidad asentó y aún se mantienen (TENTI FANFANI, 2006). No obstante una paradoja se presenta cada vez que una sociedad desatiende la educación, a nivel de una política de estado, con paupérrimos presupuestos para el sostenimiento de la infraestructura material; las posibilidades de acceso, permanencia y egreso; ciertos estándares de profesionalismo como lo son salarios y posibilidades de carrera en la profesión, entre otros requerimientos.

En Argentina, la dirección dada a la educación a partir de 2003 ha ido revirtiendo algunas de las características de identificación propiamente neoliberal que fueron erigidas en la década de 1990, como lo fue el desplazamiento de la responsabilidad hacia la esfera e iniciativa privada y organizaciones de la sociedad civil. El signo mas claro dado al respecto es la asignación presupuestaria que desde 2003 se a elevado hasta llegar a 6,47\% del PBI en 2011. No obstante muchas de las transformaciones realizadas pueden parecer un paso en discordia a la inercia de la marcha sin rumbo, que la educación venía trazando a lo largo del siglo XX, con ajustes, a modo de retazos, a la ley de educación liberal de 1884. Podemos afirmar, y asumir aquí los riesgos de ser taxativos, que las transformaciones más importantes, desde los últimos diez años hasta el momento realizadas, son de índole superestructurales, en la organización educativa, y otras de índole macroeconómica, con incidencia más o menos directa en el sector educativo. En dicho sentido son atendidas 
por un lado, cuestiones de la propuesta curricular coincidentes con el proyecto económico, social y cultural; la organización y articulación de los diferentes niveles educativos; la expansión de diferentes especialidades ó ámbitos de atención educativa. Por otro lado condiciones de acceso, permanencia y término de la educación, que atraviesan al educando y su núcleo familiar con los planes de asistencia económica ya conocidos.

Como presenta la objeción más arriba, pero ahora en forma de interrogante, concierne preguntarnos si toda transformación que pretenda ser realizada en educación podrá prescindir de la revisión y transformación de la formación, acceso, ejercicio, capacitación y progresión de la docencia; pilar estructural de la obra educativa. Profesión que se erige en torno al saber enseñar, forjado a los condiciones de las culturas, sociedades y en sus tiempos, períodos y épocas.

Si han sido tenidos en cuenta a nivel de la política nacional para la educación estos asuntos, ha sido a través de los programas y convocatoria de Investigación Pedagógica "Conocer para incidir sobre las prácticas pedagógicas" que el Instituto Nacional de Formación Docente viene reeditando desde 2007 a la fecha. Aunque hasta el momento, dicho programa de cuenta de la revisión y estudio y poco sabemos de su aprovecha miento en términos de propuestas superadoras. Es en el contexto de dicho programa, que fue realizada una investigación entre el 2010 y 2011 en un instituto de formación docente en el área de la Educación Física en la provincia de Córdoba, y sobre los hallazgos producidos se pretende extender aquí una reflexión.

Puntualmente, se propone vincular algunas transformaciones curriculares que se han dado recientemente con respecto a la formación de profesores y algunas repercusiones que pueden inferirse, podrán tener en el carácter profesional con que se desarrolle el ejercicio docente. Y estas vinculaciones son posibles de hacerse dado que las transformaciones curriculares inciden 
en la noción y concepto sobre el saber enseñar, que modela al ejercicio docente, especialmente en el espacio asignado a la formación didáctica.

Seguidamente expondremos una caracterización de la transformación curricular, los hallazgos de la investigación desarrollada y finalmente las vinculaciones entre ambas y sus repercusiones en el saber enseñar.

\section{La transformación curricular de los institutos superiores de formación docente y el espacio de la formación didáctica}

La formación de docentes en Argentina, desarrollada, en su gran mayoría, en los Institutos Superiores de Formación Docente (ISFD) dependientes de las distintas jurisdicciones provinciales, viene siendo transformada por las políticas nacionales para la educación superior. La Ley de Educación Nacional 26.206, la creación del Instituto Nacional de Formación Docente y el Plan Nacional de Formación docente, constituyen el marco, en el que junto con otros programas, se desarrolla una transformación curricular, transformación de los planes de estudio, en los referidos institutos.

En el desarrollo de la transformación curricular, las jurisdicciones provinciales, según los casos, han adaptado las recomendaciones nacionales según consultas a actores y representantes de las instituciones educativas de su ámbito. Tal ha sido el caso de los ISFD en el área de Educación Física, de la Provincia de Córdoba, que transita actualmente por la implementación del cuarto y último año del nuevo plan de estudios, iniciado en marzo de 2010. En un instituto con dichas características fue desarrollada la investigación titulada "Didáctica de la Educación Física, los sentidos asignados en la 
formación del profesorado". La atención se centró en la Didáctica de la Educación Física, concebida en el nuevo plan de estudios como una asignatura, implicando un componente curricular novedoso con relación a planes de estudio que antecedieron.

Una de las características que posiciona dichos saberes como novedosos, según los documentos oficiales de jurisdicción nacional, ha sido la distinción de formatos ó modalidades de unidades curriculares, pero con diferentes alcances. Entre ellos se encuentran el "taller", el "seminario" y la "asignatura". Ésta última en el sentido académico más estricto sería aquella unidad curricular proveedora de marcos conceptuales referenciales sólidos para la formación. Ésta particularidad de la nueva propuesta curricular, ha afectado de manera disímil a la Didáctica de la Educación Física (DEF). Inicialmente, la DEF es sugerida como un eje organizador de unidades curriculares en los documentos de la nación; y como un espacio según la modalidad asignatura, en la jurisdicción Córdoba, esto es, destacando su condición de ser una disciplina de estudio con marcos referenciales de cierta solidez. Aunque, una lectura más detenida sobre los mismos documentos y el estudio sobre el desarrollo curricular, indican cierta ambigüedad, hasta podríamos decir una controversia al momento de comprender, hasta que punto se trata de una novedad la DEF.

Parte de la controversia y que denota la asignación de diferentes sentidos dados a la DEF fue su vinculación con los espacios curriculares denominados Práctica Docente. Para algunos la DEF no habría significado ninguna novedad puesto que la Didáctica de la Educación Física habría estado alojada en la Práctica Docente, resultando el nuevo plan de estudio en apenas un desalojo del contenido didáctico, que tendría domicilio propio, ahora, como una asignatura.

Dada la relevancia de la vinculación entre la DEF y la Práctica Docente es necesario ampliar la explicación, ya que se presenta 
en ella un nudo de significación enlazado con los cambios curriculares suscecivos anteriores, que fue necesario rastrear para comprender los sentidos asignados a la DEF.

La retrospectiva nos lleva al plan de estudio que tuvo vigencia entre 1980-2000, que contenía la asignatura "Didáctica Especial y Práctica de la Enseñanza” (DEPE). Dicha asignatura estaba presente en tercer año, como DEPE I, y en cuarto año, como DEPE II, encargada cada una de ellas de los diferentes niveles de destino; primaria y secundaria, respectivamente. Cabe aclarar que, en dicha denominación no hay alusión directa a la Educación Física. La situación cambió radicalmente cuando en el plan que tuvo vigencia entre 2001 y 2009, hubo modificaciones sustanciales en el área de las prácticas. Inicialmente la asignatura pasó a denominarse "Práctica Docente" desapareciendo la partícula "Didáctica Especial". Se agregó carga horaria a las prácticas, ampliándose las prácticas docentes de primero a cuarto año, contando con práctica I, II, III y IV. Cabe el interrogante sobre cual destino le deparó al contenido sobre didáctica especial en dicho cambio. Inicialmente habría tenido dos destinos: 1) los espacios de la formación específica, que en el plan de estudio de 2001 pasaron a denominarse por ejemplo "Deportes y su enseñanza" ó "Gimnasia y su enseñanza"; 2) su conservación en el mismo espacio de práctica, a pesar de ser excluido desde la denominación dada. Aparentemente habría tenido los dos destinos.

El nuevo plan de estudio vigente, desde 2010 a la fecha, ha trazado algunas transformaciones, pero también algunas continuidades. Especialmente con referencia a las prácticas docentes. La continuidad se presenta en el sentido que se mantiene la estructura de cuatro años de práctica docente. Pero la novedad se ha hecho presente en el sentido de que la partícula "didáctica" recuperó presencia en el nuevo plan, de manera inusitada. 


\section{Didactización del plan de estudio}

El análisis de la propuesta curricular, seguido de entrevistas, y de observaciones participantes en reuniones de coordinación académica; las relecturas, interpretaciones y reinterpretaciones fueron la base para categorizar al desarrollo de la transformación curricular que se estaba observando, como didactización. Dicho proceso, la constitución del mismo, nos permite ir aprendiendo como el saber enseñar a la vez que cobra cierta relevancia, también se presenta como una imagen bastante difusa.

Con características totalmente opuestas al plan de estudio del 2001 en que el término didáctica desapareció, en el nuevo plan aparece diseminado en diversidad de espacios curriculares, formatos y áreas.

El área de formación pedagógica del viejo plan incluía el espacio curricular "Enseñanza y Currículum" y sustituyó a la "Didáctica General" del anterior, que vuelve a estar en el del 2009. Este último plan denominó, al área que lo contiene como Formación General y Pedagógica.

Las unidades curriculares de la formación específica que en 2001 incluyeron la partícula y su enseñanza, como por ejemplo "Gimnasia y su enseñanza" y que anteriormente no tenían ninguna alusión al respecto, en el plan del 2009 la perdieron, aunque algunas incluyeron la partícula "didáctica", y pasaron a denominarse por ejemplo, "Didáctica I de los Deportes Colectivos" Esta unidad se presenta en el área de la Formación Específica pero, en el formato seminario, propuesto para ser trabajados en forma conjunta por unidades curriculares con el formato taller, que trabajan separadamente dos modalidades de deporte colectivo diferentes, como por ejemplo handbol y fútbol.

La tercera forma de aparición de contenidos referentes a la formación Didáctica, finalmente es en el formato asignatura, 
perteneciendo al área específica, con la denominación de "Didáctica de la Educación Física".

En síntesis, el proceso de diseminación por todo el plan de estudios de contenidos con connotación didáctica, lo denominamos didactización. Pero dicha interpretación es válida, solo en términos de currículum prescripto. Ya que mientras que en una primera interpretación se identificó que se desarrollaba un proceso de didactización progresiva, en los planes de estudio subsecuentes a partir del currículum prescripto; una interpretación más densa indicó que la didactización no se desarrollaría según la prescripción curricular lo indicaría, sino que en se trataría más de un proceso aparente. En el proceso del desarrollo curricular, en el que fue explorado el pensamiento didáctico de los profesores de la institución investigada, se presentaron algunas inconsistencias.

\section{Didacticismo}

Una de las inconsistencias entre la prescripción y desarrollo curricular se presenta al verificarse la persistencia de considerar el contenido, por parte de algunos profesores de los espacios de la formación específica, mas como una sesión de entrenamiento que el tratamiento de la temática de la enseñanza de la modalidad deportiva en cuestión. De esta forma se presenta como bastante relativo el primer avance de didactización de la formación, que agregó la partícula "y su enseñanza" a la denominación de los espacios curriculares de la formación específica, ó en el segundo avance, en que dicho contenido forma parte de una unidad del programa, a pesar de haberse extinguido la alusión a contenido didáctico, en la denominación del espacio curricular. 
Otra inconsistencia se presentaría al relevar la articulación e integración entre todos los espacios de tratamiento didáctico, caracterizándose más como un proceso fragmentario que como un estudio sistemático. Este fue el caso de las dificultades reales encontradas entre los profesores de la formación específica, para trabajar en forma conjunta los seminarios de Didáctica I de los Deportes Colectivos, que no se pudo concretar como fuera prescripto. Otro caso es, ya desde la prescripción, que dicho espacio en el que se presenta una síntesis explicativa, se argumenta ser dicha didáctica un desprendimiento de la Didáctica de la Educación Física. Pero, paradójicamente, no se encuentra en un apartado de recomendaciones para el trabajo integrado con otros espacios, la propia $D E F$ con la cual se enuncia estar estrechamente ligada.

Con base en lo anterior, conduce a sostener una tercera inconsistencia, la cual sería, la concepción y sentido que se le asigna a la Didáctica de la Educación Física en el nuevo plan de estudios. La controversia quedó asentada en el uso de un pseudónimo al referirse a la DEF por parte de los profesores de la institución, llamándola alternada ó indistintamente Didáctica Especial ó Didáctica Específica. Según el estado de la discusión disciplinar, se trata de dos entidades diferentes, dos configuraciones del campo disciplinar didáctico diferentes, discusión disciplinar que esbozaremos mas adelante, pero al parecer poco saldada en el conjunto de los profesores que trabajan contenidos didácticos de la formación.

Con base en dichas inconsistencias se puede afirmar que el avance en la didactización de la formación no sería tal. Entiéndase, por didactización no solo la proliferación de contenidos didácticos sino también, a la organización didáctica de la formación, según criterios didácticos que interviniesen como una mirada especializada para estructurar la carrera según unidades curriculares, temas, asuntos y contenidos 
didácticos con cierta progresión y profundización. $\mathrm{Al}$ contrario, la diseminación de temas didácticos caracterizada por fragmentación y superposición es lo que más induce a identificarlo como un proceso aparente.

En contrapartida, el desarrollo curricular vendría infligiendo una especie de sometimiento en la asunción de una identidad didáctica, aunque dicha identidad no es asumida ó lo es en forma aparente. como fue caracterizado en el proceso en que un fragmento del saber específico de la Educación Física, como Deporte, Gimnasia, etc., adosó la partícula " su enseñanza" aunque los profesores del mismo continúan, aún hoy, desarrollando un proceso de entrenamiento y no de problematización de la enseñanza de deporte. En dichos términos al proceso de desarrollo curricular estudiado preferimos denominarlo didacticismo.

Para comprender el significado que damos a didacticismo, recurrimos a Bracht (1997, p. 27), quien caracterizó al pedagogicismo como la aparente adhesión de la Educación física a valores considerados pedagógicos, pero sin plantear acciones consecuentes con los mismos, sino como efectos secundarios deseables. Se refiere a la importancia pregonada y dada sobre las prácticas deportivas con relación al trabajo en equipo, valores de juego limpio, etc., sin observar las acciones efectivas desarrolladas. En nuestro caso, el didacticismo se explica de manera similar, cuando se asume denominación y contenido didáctico, pero en forma aparente.

Las consecuencias que pueden inferirse a partir de estos procesos, son poco auspiciosas para la formación de los profesores de Educación Física, y en partida doble. Por un lado, porque el desarrollo curricular presenta una forma tan distorsionada y poco progresiva en el estudio del saber enseñar, que dificulta su aprendizaje. Y, por otro lado, presenta una forma distorsionada del saber, del saber específico, del saber 
de la Educación Física, al asumir los espacios de la formación específica una identidad didáctica que presume prescindir de la identidad teórica, epistemológica, y de cualquier forma de validación de los conocimientos de la materia.

Tanto la validez del conocimiento y el de una didáctica que lo enseñe serían efectos secundarios deseables, y podrían estar antepuestos prácticas legitimadas solamente por la tradición. Tal es el caso, por ejemplo, de la enseñanza de los deportes según una metodología analítica pulverizada de técnicas motoras, que es mas una práctica ritual que viene demostrando su aferrada persistencia, pero no por ello un conocimiento válido y valioso (BRACHT, 1996, p. 87).

De acuerdo al frágil estado epistemológico de la Educación Física, que se manifiesta al momento de explicar la estructura lógica y epistemológica de los saberes constitutivos de su área, induce a suponer que la asunción de la partícula didáctica, por los espacios de la formación específica, podría suplir una justificación curricular epistemológica por una justificación didáctica. En otros términos, la razón de ser en el currículo no sería en sustancia la Gimnasia en sí, por ejemplo, sino, una determinada forma de enseñanza de la gimnasia.

La particular justificación, paradojicamente, atenta tanto contra el estatus epistemológico, como contra el estatus didáctico de la Educación Física ${ }^{1}$. En el primer caso, porque al retirar el foco del conocimiento, en las materias específicas, se podrían tratar saberes de cuestionable validez, saberes advenidos de la tradición, de mitos, etc.; y en el segundo caso, porque una didáctica específica de tal o cual contenido, solo es tal si se tiene una claridad sobre la estructuración lógica y conceptual de la materia a enseñar. 


\section{El saber enseñar en el campo didáctico-pedagógico}

La existencia de procesos curriculares que denotan didacticismo en la formación docente no debiera sorprender al estudioso atento del status del campo didáctico-pedagógico y sus vaivenes más recientes. Aunque su constatación y la producción de algunos indicadores para su identificación es un producto de la investigación realizada.

Los vaivenes del campo didáctico-pedagógico (DIAZ BARRIGA, 1998) se encuentran plasmados, ejemplarmente en el plan de estudio de 2001 en el que desapareció la denominación "didáctica" de cualquier espacio curricular, en parte fue un reflejo del abandono del cual venía siendo objeto la didáctica en los treinta años que lo antecedieron. Abandono operado por la psicologización de la didáctica, que centrándose en el saber aprender, se ciñó mas a una teoría de la instrucción, sostenida por las mas variadas escuelas psicológicas conocidas; y por el desarrollo de los estudios socio-críticos sobre el currículum, que se abstuvieron de toda pretensión prescriptiva, normativa, instrumental y técnica sobre el saber enseñar. La primera con una concepción muy reducida del acto de enseñar, con relación a la complejidad del proceso educativo; la otra con una pretensión mas centrada en el como es, en términos de currículum real y distante del deber ser sobre la enseñanza, característicamente pedagógico. Ambas fueron a su vez una alternativa al sesgo formal y normativo de la didáctica.

El saber enseñar, por tanto, ha estado atravesado por los vaivenes de estas dos tendencias y desde ambas tendencias se han formulado salidas progresivas, a dicha coyuntura del campo didáctico-pedagógico. Desde el interés mas instruccional, y con anclajes en la teoría cognitiva, se han desarrollado las didácticas específicas que con base en las problemáticas de enseñanza de 
los conocimientos de las asignaturas, reconfiguran el campo didáctico pedagógico, al integrarse y complementarse con la Didáctica General, pero sosteniéndose con un grado de autonomía, con relación al normativismo didáctico. Los estudios de origen sociológicos que dieron un pequeño viraje y complementación, con la perspectiva antropológica, indagan los escenarios escolares, las culturas que habitan en ella y permiten la lectura de la intencionalidad y de los sentidos e asignados a la educación, por sus protagonistas, trazando un atajo dilógico al sentido unidireccional que pudo tener la pedagogía.

La transformación curricular estudiada, en cuanto al currículum prescripto, denota injerencia de las dos tendencias progresivas enunciadas. La injerencia socioantropológica abrió lugar a una unidad curricular denominada, "Problemáticas Socio antropológicas de la educación" en primer año en el plan de estudio; y la injerencia de las didácticas específicas dio origen a los seminarios de "Didáctica de los Deportes Colectivos" en segundo y tercer año.

No obstante, se presentan las inconsistencias ya mencionadas. En parte, podemos suponer, que dichas inconsistencias se den a partir de que las salidas progresivas apenas si están asentadas, aún; y además, conviven con algunas tendencias que podríamos llamar regresivas. Las tendencias regresivas estarían siendo abonadas por un pesimismo teórico, propio del declino didáctico, que exaltó la dimensión práctica ó más pragmática de la enseñanza, abdicando de cualquier explicación que tuviera demasiadas pretensiones de generalización sobre el saber enseñar, como lo es el estudio razonado de una teoría de la enseñanza. De esta forma se reserva la enseñanza a una dimensión tan personalizada para el profesor que prácticamente parece prescindir de cualquier forma de discusión y revisión pública.

Algunas expresiones a los entrevistados en la institución pusieron de relieve esta comprensión al utilizar el término 
"didáctica" como un sinónimo de enseñanza y en otras expresiones que personalizaban la enseñanza en forma radical. Una expresión de uso, a ese respecto, es que "cada docente tiene su propia didáctica”.

\section{El saber enseñar, como una política de estado}

Luego de las relaciones encontradas aquí, entre la transformación curricular en la formación docente y el lugar que ocupa el saber enseñar, algunas consideraciones merecen reflexión, para pensar en la promoción de una política de estado, que impulse una progresión en la carrera docente; progresión de la educación.

La dilación sobre el estudio del saber enseñar que el didacticismo refleja, puede entenderse como la contracara de todo ardid publicitario sobre la promoción de la calidad educativa. Si la educación, reniega del desafío de enseñar cada vez mejor, de estudiar, explorar, implementar, reconstruir, rediseñar, en síntesis, de cultivar el saber enseñar, la estafa educativa es como la de cualquier campo ú ocupación que contraría su contrato social. Ya sea el médico que no respete su juramento hipocrático, ya sea el abogado que viola el sigilo de información sobre la causa del defendido.

Quizás el cultivo de la pura vocación de enseñar ancorada en la versión más personalista y antididáctica, haya sido una estrategia de supervivencia en tiempos de auténtica pérdida de rumbo educativo. Pero el letargo en dicho estado significa el detenimiento de la labor educativa. El saber enseñar y el enseñar cada vez mejor, no es una obra individual, ni personal, sino que se objetiva en teorías, técnicas, procedimientos, en casos, en situaciones de enseñanza que demandan estudio 
y comunicación. Y necesita del esfuerzo personal, del involucramiento institucional y de una progresión de carrera que lo instituya.

El ejercicio de la docencia en los diferentes niveles de la educación en la Provincia de Córdoba está regulada por el decreto ley 214 de 1963 que fija el Estatuto del docente. Y podemos preguntarnos si el saber enseñar de aquel entonces nada ha cambiado con relación al presente. Si las teorías y las prácticas del saber enseñar de aquel entonces son aún las mismas? ¿Si los requerimientos sociales sobre el enseñar continúan los mismos? Y radicalizando los interrogantes nos preguntamos ien que medida el estatuto docente está organizado por las necesidades del saber enseñar actual?

Dichos interrogantes planteados desde una pretensión profesional del labor docente pueden no encontrar oídos en la docencia establecida y moldada en la modorra de la rutina, con la mirada nostálgica de que la docencia en otro tiempo fue mejor.

\section{Bibliografía}

ACHILLII, E. Investigar En Antropología Social. Rosario: CeaCu, 2005.

ARGENTINA. Ley de Educación Nacional no 26.206. Boletín Oficial número 31.062, del 28 de diciembre, 2006.

. Ministerio de Educación. Presidencia de la Nación. Lineamientos

Curriculares Nacionales para la formacón docente inicial. Buenos Aires, 2007.

Aires, 2007.

. Plan Nacional de Formación Docente. Buenos 
Políticas Estudiantiles. Documento Marco.

$\mathrm{De}<$ http://www.me.gov.ar/infod/documentos/marcopoliticas2009. pdf>. Buenos Aires, 2007.

__ _ _ Recomendaciones para la elaboración de diseños curriculares. Profesorados de Educación Física. Buenos Aires, 2008.

BERGER, P. L.; LUCKMANN, T. La Construcción Social de la Realidad. Buenos Aires: Amorrortu, 2005.

BOLIVAR, A. Conocimiento Didáctico Del Contenido Y Didácticas Específicas. En: Profesorado: Revista de currículum y formación del profesorado, Granada, n. 9, p. 1-39, 2005.

BRACHT, V. Educación Física y Aprendizaje Social. Córdoba: Ed. Velez Sarsfiel, 1997.

CANDAU, V. M. Rumo a uma nova Didática. Petrópolis: Vozes, 1988.

CHEVALlARD, A. La Transposición Didáctica. Buenos Aires: Aique, 1996.

CONTRERAS D. A. Violencia simbólica y su impacto en el sujeto que aprende

Educación Física. Ponencia presentada en Congreso 60 Aniversario IPEF, Córdoba, Argentina, 2006.

CONTRERAS J. Enseñanza, Curriculum y Profesorado. Madrid: Akal, 1990.

DANGUISE, J.; RIOS, G. Práctica Pedagógica una nueva mirada: de lo simple a lo complejo. Córdoba: Centro editor de Córdoba, 1997. 
DAVINI, M. C. Conflictos en la evolución de la didáctica. La demarcación entre la didáctica general y las didácticas especiales. In: CAMILLONI, A. y otros. Corrientes didácticas contemporâneas. Buenos Aires: Paidos, 1996.

DIAZ BARRIGA, A. La investigación en el campo de la Didáctica. Modelos históricos. Perfiles Educativos, México: UNAM, n. 79/80, Enero-Junio 1998.

FURLAN, A. Aportaciones a la didáctica de la educación superior. Iztacala: ENEPIUNAM., 1989.

GIMENO SACRISTAN, J. La Enseñanza: Su Teoría Y Su Práctica. Madrid: Akal, 1989.

GIRALDES, M. Didáctica de una cultura de lo corporal. Buenos Aires: El Autor, 1994.

GOBIERNO DE LA PROVINCIA DE CÓRDOBA. Diseño Curricular. Profesorado de Educación Física. Ministerio de Educación, Córdoba, 2009.

GOMEZ, J. La educación física en el patio. Buenos Aires: Stadium, 2002.

GUBER, R. El Salvaje Metropolitano. Buenos Aires: Paidos, 2004.

GUILLEN DE REZANO, Matilde. Didática Especial. Buenos Aires: Kapelusz, 1966.

HABERMAS, J. Conciencia moral y acción comunicativa. Barcelona: Península, 1991.

HERNANDEZ ALVAREZ, J. Teoría Curricular y didáctica de la educación física. In: Didáctica de la educación física: una perspectiva crítica. Madrid: Biblioteca Nueva, 2003. 
NEGRELLI, J. M. Agir Metodológico, Metodología de Ensino da Eduação Física, Método de Ensino. In: Dicionario Crítico da Educação Física. Ijui: Unijui, 2005. p. 22, 274-277.

NÉRICI, Imídeo Giuseppe. Metodología do Ensino. São Paulo: Atlas, 1989.

OSORIO, F. El Sentido Y El Otro. Un Ensayo Desde Clifford Geertz, Gilles Deleuze Y Jean Baudrillard. Cinta De Moebio, n.4. Chile: Facultad de Ciencias Sociales. Universidad de Chile, 1998. Acc. <http://www. captura.uchile.cl/bitstream/handle/2250/13469/El_sentido_y_el_otro. pdf? sequence $=1>$.

REMEDI, E. Construcción de la Estructura Metodológica. Iztacala: ENEPI-UNAM, 1989.

SCRIBANO, A. Teoría Social Y Hermenéutica. Buenos Aires: Centro Editor De AméricaLatina, 1994.

ROMERO CEREZO, C. Delimitación del campo didáctico de la Educación Física y de su actividad científica. rofesorado: Revista de currículum y formación del profesorado, Granada, v. 11, n. 2, p. 1-45, 2007.

SHULMAN, L. S. Paraigma Y Programas De Investigación En El Estudio De La Enseñanza: Una Perspectiva Contemporánea. En La Invetigación De La Enseñanza. Barcelona: Paidos, 1989. p. 9-91.

TENTI FANFANI, E. Sociología de la Educación. Buenos Aires: Universidad Nacional de Quilmas, 2006.

WAINERMAN, C.; SAUTU, R. La trastienda de la investigación. Buenos Aires: Editorial De Belgrano, 1997. 


\section{Notas}

* Dan título a este artículo algunos de los hallazgos de la investigación realizada entre 2010 y 2011 titulada Didáctica de la Educación Física, la asignación de sentidos en la formación del profesorado. Financiada por concurso, en la convocatoria de proyectos de investigación, Conocer para incidir sobre las prácticas pedagógicas, por el Instituto Nacional de Formación Docente del Ministerio de Educación de la Nación, Argentina.

${ }^{*}$ Profesor de Educación Física y Magíster en Ciencias de la Educación. Profesor del Instituto Provincial de Educación Física de Córdoba, Argentina, de Didáctica de la Educación Física y Didáctica General. E-mail: <jmnegrelli@ hotmail.com>.

${ }^{1}$ Hernandez Álvarez (2003), minimiza la importancia de esto. 